\title{
Utilizing Proton Resonance Frequency of Isotopes Materials for Ultra-Precise Temperature Measurement: A Review
}

\author{
Monis Abdulmanan Abdullah ${ }^{1, *}$, Thar M. Badri Albarody ${ }^{1, \dagger}$, and Puteri Sri M. Bt Megat \\ Yusoff $^{1}$ \\ ${ }^{1}$ Department of mechanical engineering, Universiti Teknologi PETRONAS, Malaysia
}

\begin{abstract}
High energy management in nuclear system and refractory metals productions are equipped with challenging procedures in terms of precise and remote controlling. In order to predict occurrence of contamination and avoidance of huge damages, there are often difficulties to access the equipment during their operation. In addition, estimating the precise and remote nucleation critical temperature of decay and growth of radioactive materials in the nuclear system has also proven to be a great challenge. Other than that, the eutectic crystallization temperature of the refractory metals during production also need to provide a precise estimation. However, it has been understood that the conventional temperature sensors are yet to be applicable to work precisely in such harsh environments. On the other hand, proton resonance frequency thermometry phenomenon have not been utilized or developed to serve as temperature sensors; despite the fact that they are capable to measure temperature in quantum level. Therefore, this article provides a review of the prior art on proton resonance frequency thermometry with its application and reliability, and elaborates on the trajectory of ultra-precise temperature measurement as the latest development.
\end{abstract}

\section{Introduction}

Temperature measurement for any system provides crucial information about how the system performs, how far the system has been under control, what is the temperature distribution in the whole system; despite the system's size or complexity. Nowadays, the available advanced technology of temperature measurements techniques at a level of sophistication accuracy have yet to be fulfilled by many applications. In order to measure the temperature in ultraprecise manner; as required by the quantum level of the system, there is a need to understand how the available techniques proven to be precise and remotely handled, can be implemented successfully. The solid-state nuclear magnetic resonance (NMR) technique is able to provide an ultra-precise temperature measurement through the proton of particular nuclei of specific

\footnotetext{
* Corresponding author: monis g03669@utp.edu.my

† Corresponding author: dher.albarody@utp.edu.my
} 
materials [1]. This paper aims to focus on temperature measurements using solid-state NMR technique, which has a real-time capability without any ionizing radiation occurrence.

\section{Overview}

NMR thermometry techniques are divided in to two main approaches: (1) the spin relaxations measurements $\left(T_{1}, T_{2}\right)$, and (2) the chemical shifts through the magic angle spinning (MAS). Both techniques are based on the temperature dependence of the proton resonance frequency [1]. However, the fundamental relation of nuclear magnetic resonance is the proportionality between the angular resonance frequency of the proton $\omega$ and the magnetic-flux density $\beta, \omega$ $=\beta \gamma$, where $\gamma$ is the gyromagnetic ratio that nucleus specifies [2]. To make use of NMR thermometry isotopic chemical shifts of specific materials is where to begin with. Those materials are ${ }^{1} \mathrm{H}[3,4]{ }^{13} \mathrm{C}[5-7],{ }^{15} \mathrm{~N}[8],{ }^{31} \mathrm{P}[9,10],{ }^{119} \mathrm{Sn}[11-13]$ and ${ }^{207} \mathrm{~Pb}$ [14-16]. In solidstate, NMR technique is able to observe the temperature mapping of a particular sample; which also the temperature distribution of the sample. Comparing other temperature measurement techniques with solid-state NMR technique, a study has stated that it is able to provide a temperature distribution throughout the sample, not only the surface temperature of the sample as the probe is not in contact with the sample [17]. There has been a number of applications presented in the literature that represents the solid-state NMR temperature measurement techniques. In food industries, the precise reading of temperature in real time is required to ensure accurate output of the products is obtained; as a slight increment or decrement will affect the end-product. By a spatial measurements of the spin relaxations $T_{1}$ and $T_{2}$, the temperature profile can be mapped for cooking food, dairy products, and emulsified materials (a mixture of two or more liquids that are normally unmixable) [18]. Moreover, many researchers had done studies of chemical and physical characterizations of wide range of food which are categorized in terms of the amount of fat in canning meat, the percentage of water in daily dairy food process, storage of vegetables, and fruits and many others applications. With that, without adding any tracer materials, NMR is a powerful nondestructive technique which provides a specific type of information that relates to the product characterizations of chemical components and their percentage as well. The temperature mapping of the aforementioned applications can be examined by NMR technique, as shown below in Figure 1 and Figure 2, respectively.

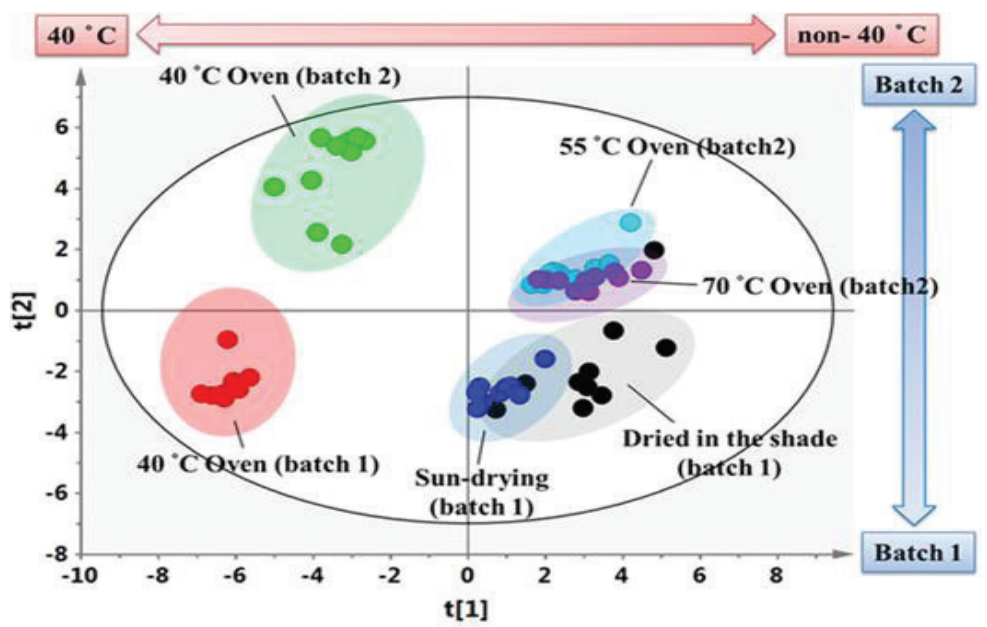

Fig. 1. Scatter plots of the PCA scores of the ${ }^{1} \mathrm{H}$ NMR dataset of extracts of Uraria Crinita that had been processed in different batches, by different drying methods, and at different oven temperatures [19]. 
(a)

(b)

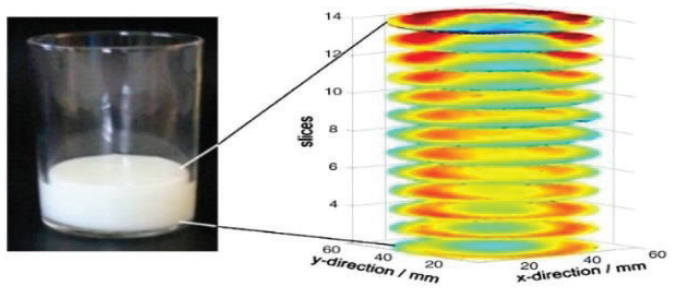

(c) MRIIR-
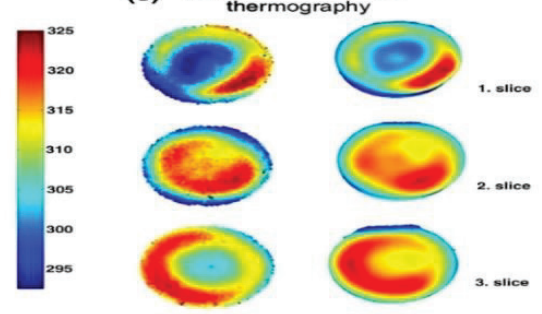

Fig. 2. (a) Heated model food with marked slices for (qualitative) comparison in (c). (b) MRI determined temperature distribution of heated model food (15 slices, slice thickness $1 \mathrm{~mm}$, slice distance $1 \mathrm{~mm}$ ). (c) Comparison of MRI and IR thermography of three selected slices [17].

However, NMR technique has been used widely in biotechnology and embryology applications. Studies on the components of biological members of humans and animals provide the exact information about their built. Doctors observed during the process of heating therapy temperatures measurement by NMR, it is able to provide directions towards where the heat should be applied and the exact amount that is required to stop additional heat, as compared with the normal tissues [20]. The temperature measurement ranges between $250^{\circ} \mathrm{C}$ to $47^{\circ} \mathrm{C}$ are identified in literature, respectively [1]. Other than that, in terms of material processing with NMR technique, there has been a wide range of application in many different industries. It is worth mentioning that the treatments process of medicine drugs is highly critical and sophisticated as it relates to individual's life. The precise temperature of syntheses of the materials is the most significant part of the process. This is due to the fact that any slight difference occurrence in the temperature can entirely change the chemical structure and provide a different output (medicine); hence leading to a side effect or inveracity of the medicine treatments [21] as illustrated in Figure 3.

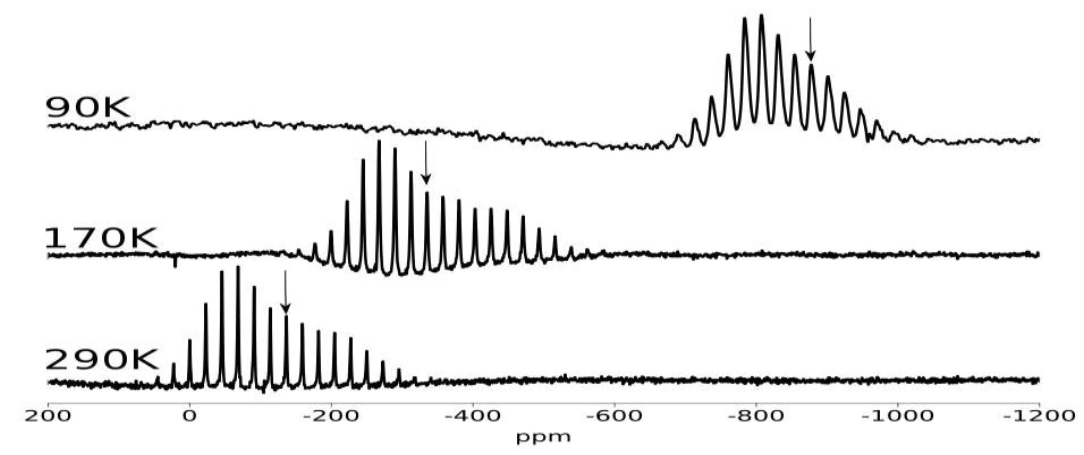

Fig. 3. ${ }^{119} \mathrm{Sn}$ MAS NMR spectra of $\mathrm{Sm}_{2} \mathrm{Sn}_{2} \mathrm{O}_{7}$ at top $90 \mathrm{~K}$, middle $170 \mathrm{~K}$ and bottom $290 \mathrm{~K}$ [22].

Moreover, the NMR temperature measurement technique has also been used in processing the steel-making while it is being accompanied with complicated multicomponent systems. NMR technique is a very powerful analysis tool to measure the 
temperature of the system and the structural analysis within all the stage of the process as well as covering a wide range of the temperatures. In situ high temperature NMR, temperature measurement could be recorded up to $1400^{\circ} \mathrm{C}$, as shown in Figure 4 . Varian infinity plus 400 (9.4 Tesla) spectrometer operating at $104.17 \mathrm{MHz}$ with a home-built static probe [23] has been used to perform in situ high temperature NMR experiments. Two different analysis techniques have been used at various temperature ranges. Relaxation time $T_{1}$, was used to measure by a saturation recovery pulse sequence and relaxation time $T_{2}$ value also calculated. It is essential to understand the fluctuations of local magnetic field with the spin lattice relaxation. However, these types of process include various types of components. Therefore, the measurement of MAS in solid state NMR plays a vital role with respect to different temperature NMR spectroscopy; once an observation of several MAS with specific line widths occurs, the data of temperature measurement is recorded. This is caused when the difference is detected due to the chemicals from one component to another component chemical shift. Each technique is complementary to the other technique, as it has to measure the temperature in ultra-precise manner by using the solid-state NMR instrument.

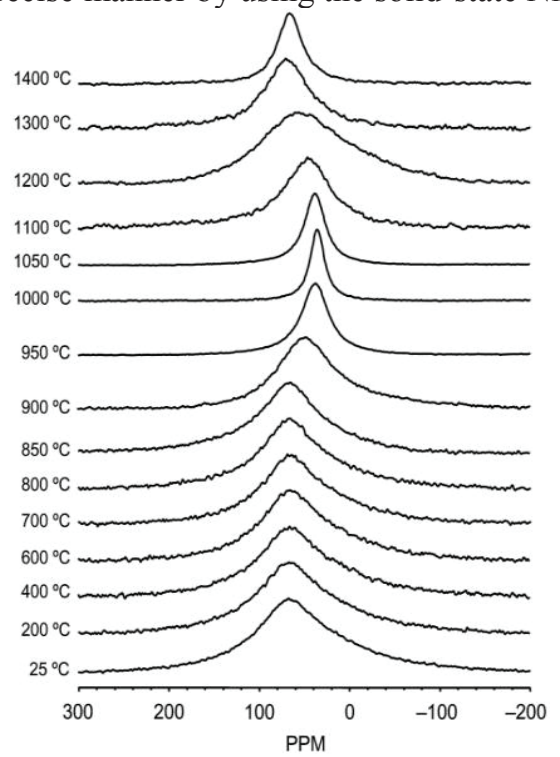

Fig. 4. ${ }^{27} \mathrm{Al}$ static spectra for the CAS glass and melt from $25^{\circ} \mathrm{C}$ to $1400^{\circ} \mathrm{C}$ [24].

Furthermore, the requirement to measure the temperature in NMR technique is not easy task in terms of acquisitions data and analysing it from the NMR spectroscopy. To this date, there is no standard formula for NMR spectroscopy data for temperature base. Many researchers have used different mathematical model techniques. Starting with a liner behaviour, the equation below shows the actual shift versus temperature relations [25].

$$
\begin{gathered}
\delta_{\text {peak }}(T)=-\{3670.6 \pm 1.0 \mathrm{ppm}\}+\{0.666 \pm 0.003 \mathrm{ppm} / \mathrm{K}\} \mathrm{T} \\
\delta_{\text {iso }}(T)=-\{3713.9 \pm 1.0 \mathrm{ppm}\}+\{0.758 \pm 0.002 \mathrm{ppm} / \mathrm{K}\} \mathrm{T}
\end{gathered}
$$

The above two empirical equations vary due to the change of isotropic and the anisotropic components of the chemical shift. Equation (1) fits anisotropic components while, the equation (2) is comparatively based on the express of the temperature in an isotopic component. The uncertainties in these equations result from a slight linear fit of the data and reflects for the high signal-to-noise ratio of the spectra. Equation (1) provides the basis for measuring temperature in a static probe because the peak can be easily determined while 
ensuring its accuracy in a limited number of scans without recourse to fitting or calculation [26]. Many other researchers have focused their studies on measuring the temperature variation by NMR solid-stat technique with the help of a known isotopic material. One researcher selected $\mathrm{Pb}\left(\mathrm{NO}_{3}\right)_{2}$, because it has a strong temperature dependence of the isotropic chemical shift [27].

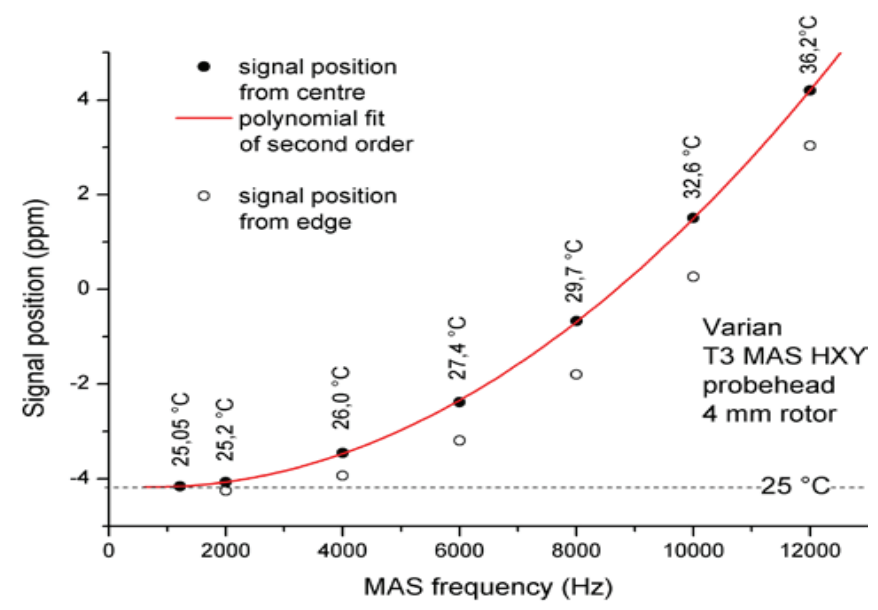

Fig. 5. ${ }^{207} \mathrm{~Pb}$ NMR chemical shifts of $\mathrm{Pb}\left(\mathrm{NO}_{3}\right)_{2}$ and the sample temperature in the center (solid circles) and edge (open circles) of the rotor as a function of the MAS rate. The measurements were carried out at VT temperature set to $25^{\circ} \mathrm{C}[28]$.

Special fitting curve has been used by the aforementioned researcher, where the secondorder polynomial point method has been utilized. Temperature gradients can be calculated from the line width of the signals of NMR spectroscopy, which indicates the temperature of the sample in the base of chemical shift (figure 5). In addition, the variable temperature measurements (VT MAS NMR) is calculated when a specific temperature needs to be measured. In NMR solid-state, a rapid high precession temperature measurements can be obtained with fluctuations of $1 \mathrm{~s}$ timescale as well as the temperature distribution that has wider ranges of temperatures of the product [29]. The authors highlighted that the demerits of this technique are very complicated in terms of result reading and analysis as it can be costly.

\section{Conclusions}

Solid-state NMR is a very powerful instrument that is utilized to analyze the chemical components of the material. Not only that, it is also able to study the physical state in spatial and temporal terms of the material at the quantum level. In addition, NMR technique does not affect the sample that is being used in the process, as it is a non-destructive testing technique. Temperature measurement of a system is remotely and instantaneously obtained in the solid-state NMR; which also covers a wide range of temperatures. Lastly, the NMR signals amplitudes and the line shapes of NMR spectroscopy has provided a meaningful insight.

\section{Acknowledgment}

The author acknowledges the support of Universiti Teknologi PETRONAS, Malaysia, for this work. 


\section{References}

1. K.R. Thurber, R. Tycko, Journal of magnetic resonance, 196, 84-87 (2009)

2. M.J. Duer, Solid-State NMR Spectroscopy Principles and Applications, Blackwell Science Ltd, USA, (2002)

3. Dvinskikh, Castro, Sandström, Magnetic Resonance in Chemistry, 42, 875-881 (2004)

4. A.E. Aliev, K.D.M. Harris, Magnetic Resonance in Chemistry, 32, 366-369 (1994)

5. A.N. Klymachyov, Dalal, Solid State Nuclear Magnetic Resonance, 7, 127-134 (1996)

6. J.F. Haw, R.A. Crook, R.C. Crosby, Journal of Magnetic Resonance, 66, 551-554 (1986)

7. G.C. Campbell, Crosby, J.F. Haw, Journal of Magnetic Resonance, 69, 191-195 (1986)

8. Wehrle, Aguilar-Parrilla, Limbach, Journal of Magnetic Resonance, 87, 584-591 (1990)

9. C.S. Zuo, K.R. Metz, Y. Sun, Sherry, Journal of magnetic resonance, 133, 53-60 (1998)

10. H. Pan, B.C. Gerstein, Journal of Magnetic Resonance, 92, 618-619 (1991)

11. T.F. Kemp, G. Balakrishnan, K.J. Pike, M.E. Smith, R. Dupree, Journal of magnetic resonance, 204, 169-172 (2010)

12. B. Langer, Schnell, Spiess, A.-R., Journal of magnetic resonance, 138, 182-186 (1999)

13. C.P., Cheetham, C.M., Journal of Magnetic Resonance, Series A, 101, 299-306 (1993)

14. T. Takahashi, H. Kawashima, H. Sugisawa, B. Toshihide, Solid State Nuclear Magnetic Resonance, 15, 119-123 (1999)

15. P.K. Isbester, A. Zalusky, D.H. Lewis, M.C. Douskey, M.J. Pomije, K.R. Mann, E.J. Munson, Catalysis Today, 49, 363-375 (1999)

16. A. Bielecki, D.P. Burum, Journal of Magnetic Resonance, Series A, 116, 215-220 (1995)

17. B. K. Knoerzer a, M. Regier b,c, E.H. Hardy d, H.P. Schuchmann b, H. Schubert, Innovative Food Science and Emerging Technologies, 10, 537-544 (2009)

18. R.R.a. Xiaofei Ye a, Paul Chen a, Kehua Chang a,I.A.T.b. Ke Ning a, Chris Doona, Journal of Food Engineering, 59, 143-150 (2003)

19. J. Chao, Y. Dai, H.Y. Cheng, W. Lam, Y.C. Cheng, K. Li, W.H. Peng, L.H. Pao, M.T. Hsieh, X.M. Qin, M.S. Lee, Scientific reports, 7, 38763 (2017)

20. M. Shigeo Okuda, $1 *$ Kagayaki Kuroda, PhD,1 Koichi Oshio, MD, PhD,1, P. Robert V. Mulkern, 2 Vincent Colucci, BA,1 Paul R. Morrison, MS,1, M. Osamu Kainuma, 1 and Ferenc A. Jolesz, MD1, Journal Of Magnetic Resonance Imaging, 12, 330-338 (2000)

21. F.C.a.S. Grzesiek*, JMB, 715, 739-752 (2002)

22. G.B. T.F. Kemp, K.J. Pike, M.E. Smith, R. Dupree, Magnetic Resonance, 204, (2010)

23. J.F. STEBBINS, 1353-1373 (1991)

24. K. Kanehashi*, Nippon Steel Technical Report, Advanced Technology Research Laboratories , 20-1, Shintomi, Futtsu, Chiba, Japan, (2011)

25. C.D.b. Guenther Neue a, Solid State Nuclear Magnetic Resonance, 7, 333-336 (1997)

26. A.C.D. Peter A. Beckmann*, Journal of magnetic resonance, 146, 379-380 (2000)

27. J. Wu, Geological And Environmental Sciences, Stanford University, (2011)

28. V. Hronský, Acta Electrotechnica et Informatica, 13, 95-98 (2013)

29. Jonathan f. Stebbins: nuclear magnetic resonance at high temperature. In modern methods in solid-state NMR: practitoner's guide, Stanford ca, (2017) 